\title{
Programas electorales y democracia representativa
}

\author{
Luz Dary Ramírez Franco \\ UNIVERSIDAD DE SALAMANCA, SALAMANCA, ESPAÑA \\ Idrf@usal.es
}

Resumen: Este documento a través de un amplio recorrido teórico, transición de la democracia a la democracia representativa, teoría de los partidos políticos, teoría de la calidad de la democracia y postulados acerca de los planes electorales defiende la tesis de que los planes electorales, dado su vínculo con los partidos políticos, contribuyen a la calidad de la democracia. La revisión teórica permite afirmar que los programas electorales son entendidos como el vínculo de comunicación entre los partidos políticos y los electores, y que los partidos están reconocidos en las constituciones como estamentos básicos para la democracia, en la cual juegan un papel clave en representación de su electorado, otorgado este por el régimen de la democracia representativa. Por tanto, los programas electorales deben recibir el mismo reconocimiento, puesto que contienen la información que ofrece respuestas a los requerimientos de los ciudadanos para que sus representantes aclaren sus acciones/ decisiones políticas. De este modo, este documento afirma que los programas electorales se vinculan al análisis del rendimiento de la democracia como variable explicativa de las dimensiones que abarcan rendición de cuentas (accountability) y capacidad de respuesta (responsiveness) como características básicas de la democracia representativa.

Palabras clave: programas electorales, calidad de la democracia, poliarquías, democracia representativa, rendición de cuentas, capacidad de respuesta

Abstract: This document through a broad theoretical path, transition from democracy to representative democracy, theory of political parties, theory of the quality of democracy and postulates about electoral plans defends the hypothesis that electoral plans, given their link with political parties, contribute to the quality of democracy. The theoretical review allows to affirm that the electoral programs are understood as the communication link between the political parties and the electors, and that the parties are recognized in the constitutions as basic estates for democracy, in which they play a role key in representation of its electorate, granted this by the regime of representative democracy. Therefore, the electoral programs must receive the same recognition, since they contain the information that offers answers to the citizens' requirements so that their representatives clarify their political actions / decisions. In this way, this document states that electoral programs are linked to the analysis of the performance of democracy as an explanatory variable of the dimensions that encompass accountability and responsiveness as basic characteristics of representative democracy.

Keywords: Electoral programs, quality of democracy, polyarchy, representative democracy, accountability, responsiveness 


\section{Introducción}

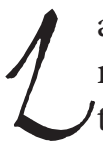

a democracia representativa o democracia indirecta, caracterizada por ser un régimen político en el cual los/las ciudadanos/as ejercen el poder político a través de sus representantes, elegidos estos por medio del sufragio universal en procesos electorales libres y periódicos, se ha extendido a todos los continentes; 183 de 196 países independientes, reconocidos por la Organización de las Naciones Unidas (ONU), llevan a cabo elecciones. En todo caso, hay que tener en cuenta que existen diferentes niveles de democratización, tanto entre los Estados como entre los agentes políticos; por ejemplo, el voto es obligatorio en 25 países (11 latinoamericanos, 7 europeos, 2 asiáticos, 3 africanos y 2 oceánicos). Debido a la propagación mencionada anteriormente, a partir de los años 90 los estudiosos de las ciencias políticas se enfocan en el estudio del rendimiento de dicho régimen político, es decir, un grupo de analistas se propone evaluar el funcionamiento del régimen y su calidad. Este compendio de estudios se caracteriza por ser heterogéneo tanto en las metodologías como en los resultados, ello debido quizás también a la diferencia entre las dimensiones que definen los indicadores que se utilizan como cuantificadores de calidad democrática, y a las fuentes de información. A causa de ello, la discusión acerca de los determinantes del rendimiento del régimen de la democracia representativa es un tema abierto y actual. En esta dirección, este artículo se une al debate defendiendo la tesis de que los planes electorales, al tratarse de un documento vinculante entre los partidos políticos y el electorado, son uno de los canales a través de los cuales los partidos influyen en la calidad de la democracia. Para ello, el artículo revisa la línea de tiempo del desarrollo de la teoría de la democracia, la democracia representativa, los partidos políticos, la calidad de la democracia y los postulados acerca de los planes electorales. A través de este análisis, se observa que el paso de la democracia a la democracia representativa implica su vinculación intima a los partidos políticos; el papel de estos dentro de las sociedades modernas queda determinado por ser reconocidos como las organizaciones de las cuales emanan los posibles representantes de los ciudadanos, y, por tanto, se procede a registrar en las constituciones de múltiples Estados que estas organizaciones son el cimiento del vínculo Estado y sociedad. A partir de este tejido teórico, este artículo defiende también que los programas electorales, definidos por la literatura de referencia como el contrato entre partidos y electores, deben tener el mismo reconocimiento institucional que los partidos. Este argumento se justifica en el hecho de que los programas contienen toda la información de la que los ciudadanos disponen para decidir su voto, para conocer los mecanismos, los presupuestos y las políticas a través de las cuales se satisfarán sus demandas, además de en que la definición y la sociabilización de estos documentos implican recursos públicos. Es decir, el artículo muestra como la teoría articula el trío partidos-programas-ciudadanos, el cual se vincula con las características del régimen político, rendición de cuentas y responsiveness, para afirmar que los planes electorales son variables explicativas de las mismas, y que, 
por tanto, los programas deben ser incluidos en los análisis de calidad de la democracia, demostrando de esta manera la tesis antes formulada. El documento concluye también que el no reconocimiento de los programas como documentos jurídicos es una señal de la falta de democratización del conjunto de actores políticos Estadopartidos-ciudadanos, porque este es un mecanismo de dinamismo de las contiendas electorales y parte integrante de la estructura definitoria de los partidos políticos, con lo que se convierte, como los partidos mismos, en pieza clave del sistema político y del régimen de representación democrática.

El estudio del tema es relevante, porque aunque tanto la calidad de la democracia como los planes electorales han sido abordados por los estudiosos de las ciencias políticas, la interrelación entre ambos temas es prácticamente nula, lo cual se explica quizás por el papel secundario que se le ha dado a los programas electorales dentro de la estructura tanto de los partidos como del sistema político, aunque también puede explicarse, como afirman algunos críticos, por el hecho de que la metodología del cálculo de la calidad de la democracia excluye características institucionales o del actuar político. Por esta razón, este documento, al buscar la interacción entre ambos temas, o bien abre la puerta a la discusión acerca de la necesidad de dar mayor importancia a los programas electorales, a las evaluaciones de la viabilidad y cumplimiento de los mismos, o a su reconocimiento jurídico, o bien amplia el conjunto de variables que explican el buen funcionamiento y, por tanto, la calidad de la democracia.

Este documento está organizado según los parámetros definidos a continuación: En primera instancia, se desarrolla la introducción correspondiente; posteriormente, en el segundo apartado, se presenta un análisis de la evolución de la democracia representativa y la teoría de los partidos políticos, con el objetivo de encontrar el vínculo entre ambos esquemas. En el tercer apartado se realiza un análisis de la evolución de la teoría de la calidad de la democracia y de los postulados acerca de los planes electorales; como en el caso anterior, el objetivo es intentar conectar ambos marcos teóricos para luego buscar la conexión entre los agentes que describe cada uno de los cuatro esquemas. En el apartado cuarto se presentan las conclusiones obtenidas por la revisión teórica, y se discuten las implicaciones de las interrelaciones de esos cuatro agentes, presentando finalmente los argumentos que nos permiten dar por cierta la tesis formulada.

\section{Democracia representativa vs teoría de los partidos políticos}

A través de la revisión necesaria del compendio literario de referencia acerca del concepto democracia (véase Tabla 1) nos encontramos con que, efectivamente, tal y como lo enuncian numerosos autores, la democracia se heredó de Grecia; se escribe y se repite que fueron los traductores de Heródoto quienes acuñaron el termino democracia para referirse a una comunidad gobernada por el pueblo (demos). También se 
escribe que la democracia Clásica no fue más que la forma de gobierno que desplazó a la monarquía griega, y que no se puede equiparar con el régimen democrático que define el sistema político actual. La democracia griega se desarrollaba en las polis, ciudades o comunidades pequeñas que ejercían de centros políticos, sociales y económicos, y, de facto, como ciudades-estado independientes, de ahí que, por esta misma razón, este tipo de democracia se aplicara de forma directa; los habitantes de la polis decidían las políticas a implementar mediante el acto de la aclamación, los cargos eran asignados por sorteo y de manera inmediata, y aunque los candidatos al sorteo eran los ciudadanos libres, en la democracia griega había exclusión por género, por posición social y por edad. La democracia actual se reseña desde la Revolución Inglesa, la Independencia Norteamericana y la Revolución Francesa, y se define como un sistema político que abarca el pueblo en su conjunto, para conseguir lo cual requiere que ese pueblo proceda a elegir unos representantes a través de elecciones legales e incluyentes, quedando los derechos y las demandas de los ciudadanos protegidos y avalados tanto por la constitución como por el estado de derecho, que es el principio regidor de la comunidad social que conforma el Estado. Además, este régimen también se caracteriza por el establecimiento de medios de control a los representantes, la rendición de cuentas o accountability, elemento que no existía en la democracia clásica, y que consiste en que los representantes (funcionarios públicos) deben informar y publicar todas las acciones que ejerzan en nombre del gobierno, $y$, en caso de fraude, faltante, o cualquier otro requerimiento, responder ante sus electores.

Dos observaciones surgen del concepto y la delimitación de ambos regímenes democráticos. Primero, hay un vacío de muchos años, incluso siglos, entre la identificación de un régimen y otro. De acuerdo con la revisión teórica, se entiende que el proceso de desarrollo de la democracia tal cual la concebimos hoy fue lento, avanzando y configurándose poco a poco en algunos Estados, mientras en el grueso de países seguía imperando la monarquía y la oligarquía. Fue con pensadores como Locke, (1689-1690 [2002]) y Montesquieu (1748 [1984]) cuando se inició el libre pensamiento y, en particular, la separación de poderes que iría dando forma al sistema democrático. De esta manera, el planteamiento de estos dos autores y de otro grupo selecto de intelectuales (véase Tabla 1), y las revoluciones antes mencionadas, sentaron las bases para la determinación tanto del poder del pueblo como de sus representantes en los órganos estatales. Segundo, el régimen político en cuestión debatía dentro de su construcción quienes eran las instituciones y órganos que podrían representar la soberanía del pueblo, y aunque desde los regímenes monárquicos-absolutistas existían grupos denominados facciones, las cuales se vieron obligadas, a partir de esos tres hechos que marcan el punto de inflexión entre la democracia clásica y la democracia representativa, a articular los intereses de los parlamentarios que sustituirían a la monarquía o a la oligarquía, no fue hasta finales del siglo XVIII cuando los partidos, tal cual los conocemos hoy, se institucionalizan y se posicionan como pieza clave para el vínculo entre 
el Estado y la sociedad del régimen político naciente. Por tanto, el análisis y la construcción de la teoría de los partidos políticos, aunque nace con el surgimiento del pensamiento político liberal, no empieza a difundirse hasta unos años más tarde, cuando estas formaciones no solo se han organizado y definido como partidos, sino que han recibido, mucho tiempo después, la aceptación de políticos, pensadores y de la sociedad en general. Es así como los primeros estudios acerca de estas organizaciones salen a la luz a finales del siglo XIX y a comienzos del siglo XX (véase Tabla 1). Dentro de ese cuerpo teórico, entre muchos otros temas, se empiezan a delimitar las funciones de los partidos políticos, pero en cualquier caso, aunque son variadas, por razones de interés y concisión, este artículo se remite a la función específica de agregación, canalización y divulgación de las posiciones y las demandas de los ciudadanos. El cumplimiento de esta función exige a los partidos la presentación de un programa que, aunque basado en su plataforma política como documento de constitución de la misma, exprese también las medidas que permitan atender las situaciones coyunturales de los países. De este modo, los planes electorales pueden definirse como el documento canalizador y cristalizador de las demandas de los votantes y las ofertas de los partidos.

Llegados a este punto, se ha encontrado un hilo conductor entre el régimen político de democracia representativa, los partidos políticos y los planes electorales. Desde un punto de vista analítico, este hilo adquiere mayor grosor si, para el vínculo entre estos tres niveles, se considera la rendición de cuentas que exige la democracia representativa a los actores políticos. Por un lado, los partidos políticos son reconocidos como sujetos de apoyo de los Estados, debido principalmente a su función de intermediarios en el papel político de los ciudadanos. En efecto, las constituciones de los países reconocen a dichos partidos como entidades básicas del sistema democrático, o como expresión del derecho de participación política de los ciudadanos a través de colectivos, grupos o asociaciones, y, en algunos otros casos, les otorgan el carácter de personas jurídicas, siendo muy pocos los países que en su carta magna no hacen mención alguna a los partidos políticos como parte del sistema institucional ni les atribuyen los privilegios mencionados. Es el reconocimiento explicado y generalizado el que obliga a los políticos a rendir cuentas de sus actos como funcionarios del Estado, porque en el mismo recorrido por las diferentes constituciones, y como complemento del reconocimiento jurídico que estas otorgan a los partidos políticos, cabe constatar que también proceden a señalar las funciones y las obligaciones de los mismos. De este modo, tanto la teoría como la institucionalidad que representa la carta magna dan a la rendición de cuentas un papel determinante para el funcionamiento y la calidad de la democracia representativa. Por otro lado, y a pesar de los argumentos anteriormente detallados, el plan electoral, que definitivamente es el vínculo o contrato entre partidos políticos y votantes, no tiene reconocimiento a nivel jurídico; sin embargo, en este punto es obligado enfatizar que a nivel teórico este reconocimiento subyace tanto en la teoría de la democracia representativa como en la teoría de los partidos. 
Tabla 1

Línea del tiempo de la democracia representativa y los partidos políticos

\begin{tabular}{|c|}
\hline $\begin{array}{l}\text { Del régimen democrático a la democracia } \\
\text { representativa }\end{array}$ \\
\hline $\begin{array}{l}\text { Democracia Griega o clásica, Siglo VI a.c.: Demo- } \\
\text { cracia directa, no representativa y sin partidos po- } \\
\text { líticos. }\end{array}$ \\
\hline $\begin{array}{l}\text { Filósofos Griegos: Discusiones acerca del Estado, la } \\
\text { nación, el régimen de gobierno, el pueblo, el poder. } \\
\text { Siglo IV y V a.c. }\end{array}$ \\
\hline
\end{tabular}

(Maquiavelo, 1532 [2010]; Bodino, 1576 [1992]; Hobbes, 1651 [1987]; Locke, 1689-1690 [2002]; Rosseau, 1762 [1998]): La Teoría del Estado.

(Mostesquieu, 1748 [1984]): La división de los poderes.

Revolución Inglesa (1688), Independencia EE.UU. (1776) y Revolución Francesa (1789): Democracia Representativa.

La Nueva Teoría del Estado (NTE), (Carl Friedrich von Gerber, 1880 [1913]); Laband, 1876 [1979]; Jellinek, 1900-1905 [1981]; Heller, 1929 [1998]): El estudio del Estado pasa del análisis político y filosófico al análisis jurista y sociológico.

Teoría moderna de la representación, (Pitkin, 1967 [1985]; Diggs, 1968; Laporta, 1989; Sartori, 1999; Bobbio 1999; Didier, 2000; Ferrajoli, 2007; Rehfeld, 2009; y Lifante, 2009): Régimen de Gobierno en el que el poder político del Estado y la soberanía le compete a todo el pueblo de manera indirecta. Esto es debido a que el poder es ejercido por unos representantes sujetos a rendición de cuentas y elegidos por procesos electorales incluyentes, periódicos y libres.

Nuevas formas o evolución de la teoría de la representación: Democracia participativa.

\section{Partidos políticos}

Análisis clásico de los partidos políticos: (Ostrogorski,1964 [1902]; Michels, 1962 [1911]; Weber, 1968 [1922]; Merriam, 1922; Schattschneider, 1942; Key,1949): Estudian el origen, el funcionamiento y el contexto en el que los partidos se mueven. En términos muy amplios, este grupo de autores identifican los partidos políticos como grupos sociales que buscan el control gubernamental. De esta parte de la teoría surge el debate acerca del papel de los partidos en el desarrollo de la democracia.

Auge del estudio de los partidos políticos: (Duverger,1954; Ranney y Kendall,1954; Neumann, 1956; Eldersveld, 1964; Sorauf, 1964; LaPalombara y Weiner, 1966; Epstein, 1967; Lipset y Rokkan,1967; Sartori,1976). A este grupo de autores se les debe la formulación del marco teórico y el consecuente análisis empírico que, además de explicar formalmente el origen de los partidos teóricos descrito por los clásicos, permite entender el sistema de partidos y las funciones de estos.

Este mismo grupo de autores, con base en el trabajo de Schattschneider (1942), desarrolla el modelo de partidos responsables, el modelo espacial de Downs (1957) y el modelo de la ley curvilínea de May (1973).

Los partidos en la actualidad: El funcionamiento de los partidos debe ser analizado considerando el impacto de las redes sociales y de la tecnología. Está claro que estos factor

es han afectado tanto a la composición de los partidos como al sistema de partidos, debido quizás a la mayor participación de los ciudadanos, a través de estos medios. 


\section{Los planes electorales vs la calidad de la democracia representativa}

El anterior análisis concluye que tanto la teoría de referencia como las premisas constitucionales mayoritariamente extendidas determinan que la rendición de cuentas es un elemento clave a través del cual los partidos políticos pueden influir en la calidad de la democracia, afirmación que dichos marcos de referencia teórico y constitucional aún no hacen extensiva a los planes electorales. Por esta razón, este apartado se plantea como objetivo prioritario presentar, a través del análisis de la literatura teórica y empírica, argumentos que permitan defender la tesis de que los manifestos, como documentos vinculantes entre partidos políticos y electores, son uno de los canales a través de los cuales los partidos pueden influir e influyen de facto en la calidad de la democracia.

Un nuevo recorrido teórico, en esta oportunidad a través de la literatura existente acerca de la calidad de la democracia representativa, a la cual es necesario sumar lo poco que se puede rescatar en términos literarios tomando como referencia los programas electorales (véase Tabla 2), nos muestra que la definición de calidad democrática implica delimitar los conceptos de democracia, poliarquía, democratización y calidad, en este mismo orden. En todo caso, antes de entrar de lleno en la conceptualización mencionada, es necesario aclarar que la gran mayoría de teóricos solamente llegan a un punto claro de consenso, y este es que todos y cada uno de los posibles términos a estudiar dentro del ámbito de las ciencias políticas son complejos o controvertidos. Este documento, respetando todas las posiciones profesionales, establece un principio básico de partida, principio que defiende que, a partir de la construcción del cuerpo teórico presentado en las Tablas uno y dos, se pueden delimitar conceptos claros, objetivos y definitorios respecto a las realidades de los temas estudiados, conceptos que, por tanto, permiten abordar discusiones también objetivas $y$, sobre todo, válidas. A partir de dicha directriz, se procede a definir cada uno de los cuatro términos mencionados anteriormente, combinando los aportes teóricos de interés sobre el supuesto de estudio, siempre sustento de las discusiones académicas.

La democracia se entiende como la forma de gobierno que da poder y soberanía al pueblo; esta forma de gobierno se desarrolla a través de elecciones libres, por medio de las cuales los ciudadanos eligen a sus representantes, sujetos estos a la rendición de cuentas.

Las poliarquías fueron definidas por Dahl (1971) para identificar las formas de gobierno de occidente como democracias incompletas, debido a que dichas formas de gobierno, aunque inicialmente cumplen los requisitos formales básicos para ser reconocidas como democracias, están muy lejos de ser gobiernos ejercidos por el pueblo. 
La democratización, por su parte, se refiere al proceso de implementación de las condiciones y la institucionalización del sistema democrático, y, en este sentido, está compuesta por las diferentes fases de la democracia. Por esta razón, y debido a la complejidad que adquiere la aplicación de la condición básica de la democracia de otorgar el poder absoluto al pueblo, la democratización no parece ser un proceso donde pueda existir una fase final o de cierre.

Por último, el término calidad, referido a la democracia, se define desde el concepto de producto delimitado por la economía (Diamond y Morlino, 2005); los autores afirman que la calidad de la democracia está determinada por el proceso, el contenido y el resultado de la aplicación de este régimen político. El proceso se refiere a la forma sistemática con que la implementación y el desarrollo de la democracia son controlados a través de la rendición de cuentas y la solidificación del Estado de derecho. El contenido concierne a la estructura diseñada para que ese estado de derecho incluya, defienda y asegure el cumplimiento de los derechos de igualdad, libertad y participación política de todos los ciudadanos. El resultado incluye el estudio del grado de satisfacción de los ciudadanos, es decir, que se evidencie reciprocidad entre las demandas de estos y el cuerpo institucional diseñado para dar respuesta a las mismas.

A partir de todo lo anteriormente explicado, se puede expresar que la calidad de la democracia debe ser entendida como el nivel de desarrollo en los Estados, en términos de democratización, de todos los agentes e instituciones, de los mecanismos que cristalizan las libertades y los derechos de los ciudadanos, de la aplicación de la normativa que permite asegurar el cumplimiento, la permanencia y la equidad de ese compendio de derechos, así como de la optimización de la respuesta, en términos de abastecimiento, a las demandas de los ciudadanos, con respecto a la satisfacción de estos. En otras palabras, la calidad de la democracia mide la organización institucional y sus mecanismos para crear espacios de participación ciudadana, y estos dos factores encierran, de acuerdo a la literatura de referencia, el conjunto de dimensiones que evalúan los componentes contenidos en los conceptos arriba descritos, y el papel de cada uno de los actores de la esfera política en el desarrollo de una buena democracia; estas dimensiones son el respeto a la ley o democratización del Estado, espacios de participación ciudadana, mecanismos de rendición de cuentas, satisfacción de los ciudadanos con respecto a sus demandas, equidad en derechos, libertad de los ciudadanos, y los mecanismos a través de los cuales se aseguran los derechos también de los ciudadanos.

Muchos han sido los autores que se han dedicado a evaluar la calidad de la democracia, y la gran mayoría se basan en la delimitación de las dimensiones pro- 
puestas por la teoría (Coppedge, 1990, 1994, 2002; Inkeles, 1990; Beetham, 1994a; Lijphart, 1999; Altman y Pérez-Liñán, 2002; IDEA, 2002; O’Donnell, Vargas Cullell y Iazzeta, 2004; Rueschemeyer, 2005; Levine y Molina; 2007; Coppedge, Gerring, Altman, Bernhard, Fish, Hicken, ... y Semetko, 2011). La metodología del cálculo de las variables que definen las dimensiones, y las fuentes de información tomadas para ello, han generado un debate acerca de si estos estudios miden realmente la calidad de la democracia o más bien la democratización. También hay quienes alegan que al no considerarse todas las dimensiones dentro de un solo estudio, no se está midiendo la calidad de la democracia al completo, sino solo parcialmente. Otro grupo de autores afirman que medir la calidad de la democracia partiendo de diferentes niveles de desarrollo de la democracia impide hacer un comparativo entre unos Estados y otros. En lo que sí coincide el grueso de los trabajos empíricos es en afirmar que la poliarquía es una condición necesaria pero no suficiente para alcanzar un buen nivel de democracia. En efecto, muchas de las investigaciones toman como punto de partida una poliarquía, y, a partir de su nivel democrático, calculan indicadores, con base en las dimensiones teóricas, que permitan afirmar que Estado está más lejos o más cerca de esa poliarquía. Vale la pena recalcar que aunque la discusión acerca de la validez de la metodología o de las variables que usan los estudios que analizan el rendimiento de la democracia representativa está abierta y presenta por parte de los ponentes fuertes críticas, este compendio de trabajos sí sirve para identificar Estados con mayor o menor democratización de sus actores, y para identificar las normativas y los mecanismos de regulación y control de las acciones de los representantes. Además, este compendio de investigaciones también ha ayudado a definir con mayor precisión algunas de las dimensiones planteadas por los teóricos. Es decir, tal y como se señaló más arriba, aunque no haya consenso y se considere que es de una complejidad importante aspirar a medir la calidad de la democracia, el esquema teórico y los análisis empíricos deben ser entendidos como una guía para identificar, como mínimo, en que países la democracia tiene un mejor funcionamiento, de acuerdo con la normativa estándar propuesta por la teoría desarrollada bajo el concepto democracia.

Con base en la revisión de la literatura teórica y empírica discutida, este artículo considera que los programas electorales son una variable que ayuda a explicar la calidad de la democracia representativa. Por ello, se plantea que estos deben estar incluidos de manera explícita dentro de las variables que explican las dimensiones que definen la accountability electoral y la responsiveness, con el objetivo de evaluar estas como características básicas presentes en las democracias representativas. La rendición de cuentas parte de la premisa de que los representantes políticos deben 
responder por sus acciones políticas, y uno de los aspectos que componen esta dimensión es el de la información. Esa información debe dar cuenta no solo del acto o la decisión que está siendo cuestionada por los ciudadanos, sino que debe sustentar el origen de aquella, el cual debería estar justificado en gran medida en el programa electoral, pues a fin de cuentas este es el documento que se presenta como propuesta de gobierno. Del mismo modo, si el origen de ese acto no está registrado en el plan electoral, deben argumentarse entonces las razones que llevaron al gobierno a poner en marcha dicha iniciativa. Estaremos de acuerdo en que para que este proceder fuera definitivamente legitimado el plan electoral debería tener carácter judicial, y, por ello, la discusión que aborda este documento se encamina en esa dirección. La Tabla dos, en su columna de la derecha, hace referencia al vínculo existente entre la teoría de los partidos políticos y los programas electorales, supuesto que puede ser reforzado con la afirmación realizada en el primer apartado respecto de que el reconocimiento de los partidos como sujetos articuladores entre el Estado y los votantes, y la definición de sus funciones y de su financiación a través de las constituciones de los países, es un mecanismo facilitador de la accountability. Por todo ello, este artículo asevera que el programa electoral, reconocido por la literatura teórica como el contrato que vincula las demandas de los ciudadanos con la oferta de los partidos en términos de economía, empleo, educación, sanidad, política exterior, medio ambiente y atención a grupos minoritarios, merece la misma institucionalidad que los partidos políticos, porque este documento es la base de la información que exige la respuesta que los representantes políticos deben dar a sus votantes cuando son consultados o requeridos. Asimismo, los partidos políticos y las campañas electorales son objeto de financiación pública, siendo claramente la elaboración y divulgación de los programas electorales uno de los objetivos principales del uso de dichos recursos públicos, razón por la cual los políticos deberían también rendir cuentas sobre esa partida presupuestaria, variable que necesariamente se debería integrar en la accountability, con el fin de evitar que dichos costes se sigan asumiendo en vano. En cuanto a la responsiveness, los Estados más democratizados cuentan con ciudadanos también más democratizados, por lo que de forma relativamente frecuente el escenario político muestra a grupos, asociaciones o ciudadanos en general intentando ejercer su derecho a exigir el cumplimiento de las promesas plasmadas en el programa electoral, pero quedándose asimismo frecuentemente con las manos vacías por el hecho de que este es aún un documento sin reconocimiento legal. Y es que, por supuesto, cualquier sociedad que esté buscando una mayor democratización de sus actores se encontrará con que estos tenderán siempre a valerse del único documento que se genera como acuerdo tácito entre votantes y partidos/gobiernos. 
La reciprocidad que mide la satisfacción de las demandas de los ciudadanos, igual que en el caso de la accountability, debe sustentarse en los programas electorales, pues son estos los que describen los recursos que los partidos destinarán a satisfacer las expectativas y las preocupaciones de los votantes. Además, en los mismos se deben referenciar las medidas que los partidos políticos adoptarán una vez ganadas las elecciones para alcanzar los objetivos que se relacionan con la búsqueda del bienestar de los votantes de dichos partidos. Si se combinaran la responsiveness y la accountability, los programas electorales deberían tener, como mínimo, una evaluación ex-ante y una ex-post, que permitieran examinar la viabilidad y los resultados, respectivamente. De hecho, la responsiveness exige, por definición, la evaluación ex-post de los actos y las decisiones tomadas por los representantes políticos para dar cuenta de la satisfacción o no de las demandas de los ciudadanos, teniendo en cuenta que, como ya se dijo con anterioridad, los fundamentos de las mismas están registrados en los programas electorales.

Mirando el análisis desde la perspectiva opuesta, en la que se plantean ambas dimensiones, y considerando la dirección representante-votante, la democratización de los ciudadanos debe conducir a que estos se responsabilicen también de dar una mayor relevancia a los programas electorales, promoviendo su carácter contractual en términos jurídicos. Es decir, el votante debe mostrar interés por el contenido de los programas electorales, dado que con ello se asegura una información clave acerca de lo que puede y debe esperar del representante político, información a partir de la cual posteriormente puede hacer valer sus derechos y hacer oír su voz en debates que conciernan a los resultados de los gobiernos, así como también fundamentar la toma de decisión de su voto futuro.

El carácter no jurídico de los programas electorales es un indicador de la falta de democratización tanto de los ciudadanos como de los partidos políticos y los Estados. De acuerdo con la teoría, la evaluación de la calidad de la democracia representativa presupone un mínimo de democratización de actores e instituciones, por lo tanto, incluir el plan electoral como variable explicativa del rendimiento de ese régimen político complementa el análisis y amplía la definición o la relativización de una democracia con respecto a una poliarquía. La academia, por medio de un grupo de investigación denominado European Consortium for Political Research (ECPR), puso en marcha a partir de 1976 una base de datos que hoy se conoce como Manifesto Research on Political Representation (MARPOR), base de datos que arroja información sobre democracia relacionando la competición partidaria y el papel del gobierno en la elaboración de política pública, permitiendo con ello la evaluación de la relación entre partidos políticos y demandas de los electores. El marco teórico 
sobre el cual se sustenta la construcción del MARPOR es la teoría de la relevancia y la teoría de los partidos responsables. La primera vertiente teórica plantea que los partidos políticos, durante sus campañas electorales, presentan con mayor énfasis los temas que ellos consideran que son sus puntos fuertes, o en los que creen tener ventaja sobre sus rivales. La segunda vertiente presenta un análisis en el cual se describe que la contienda electoral se disputa entre dos o más partidos, y que estos ofrecen a sus votantes, a través de los programas electorales, diferentes opciones de política para cubrir sus demandas. De esta manera, el programa debe ofrecer al elector información que le permita discernir claramente que las medidas planteadas satisfarán sus demandas. El partido/candidato que sale elegido se ve obligado a cumplir las promesas electorales porque el votante premia o castiga con base en el contenido del programa. El MARPOR se construye bajo la metodología de análisis de contenido de textos, en este caso, los programas electorales, a través de la cual se pueden identificar las políticas y la posición ideológica de los partidos políticos. Los planes electorales son evaluados con rigurosidad, para lo cual el MARPOR creó un sistema de codificación que permite clasificar las preferencias políticas de los partidos, las cuales son un set de registros positivos o negativos que los partidos asientan en sus programas electorales con relación al conjunto de políticas públicas, económicas sociales, de bienestar, de educación, de medio ambiente y laborales, entre otras, conducentes a atender las demandas de los electores. El MARPOR afirma que el programa electoral es válido como unidad de estudio, debido a que este es el documento que los partidos políticos presentan a los votantes como guía de sus propósitos y sus tendencias ideológicas, además de ser un documento acreditado y validado por el partido correspondiente. El MARPOR considera también que el estudio de los programas electorales facilita el análisis comparativo tanto en el interior del partido como entre partidos, debido a que estos se presentan para cada año electoral y contienen temas diversos y comunes en términos de medidas gubernamentales (Alonso et al., 2012; Ares y Volkens, 2017).

El MARPOR ha construido lo que ellos denominan "Esquema de clasificación estándar", el cual describe las preferencias políticas, la política y las políticas que son abstraídas de los programas electorales. El equipo del MARPOR amplía el esquema presentado por Robertson (1976), el cual pasa de contener 21 categorías a contener 56. Las categorías se construyen por medio de la codificación de los programas electorales presentados por los partidos ganadores de una contienda electoral, y la unidad de codificación es la cuasi-frase o parte de una frase que contenga un argumento completo. Posteriormente, cada cuasi-frase se asigna a una de las categorías del esquema, que está definido por siete grandes áreas o dominios que integran un 
número finito de subcategorías en su interior. De esta manera, el esquema representa los datos que produce el MARPOR, y que se relacionan con temas de Relaciones Internacionales, Democracia, Sistema Político, Economía, Bienestar y Calidad de Vida, Tejido Social y Grupos Sociales. La base de datos también incluye subcategorías que identifican las particularidades de las economías del centro y del este de Europa, así como el índice RILE de posicionamiento ideológico de los partidos, además de lo cual asigna a cada partido un código que lo posiciona dentro de una de las 11 familias de partidos que identifica. La base de datos también incluye información sobre datos electorales, como puede ser el porcentaje de votos obtenidos por cada partido, el porcentaje de votos en elecciones presidenciales, el número absoluto de escaños por partido y el número total de sillas en el parlamento, entre otros. El esquema está diseñado a partir de elecciones llevadas a cabo entre 1945 y 2015, considerando para ello 60 economías, de las cuales cinco son latinoamericanas, y las variables se construyen con la codificación de 4.392 programas electorales de 1.146 partidos políticos, que han participado en 741 contiendas electorales, con datos actualizados en julio de 2018 (Volkens et al., 2018). La codificación de los programas electorales se realiza de manera manual, bajo la consideración de que las personas delimitan de manera más fina que los ordenadores la interpretación de los contenidos de los programas (Ares y Volkens, 2017).

La base de datos MARPOR, debido a su minuciosa construcción y a la revisión de esta con pruebas estadísticas tales como cálculos de verificación del número de programas sin codificación, comprobación de la relación contenidos de los programas vs categorías MARPOR, entre otras verificaciones, es confiable, válida y robusta. En efecto, dadas sus fortalezas, existe un amplio compendio de investigaciones que se han valido de este recurso bien para evaluar postulados teóricos de la ciencia política, bien para realizar análisis empíricos que ayudan a entender el desarrollo de la relación electores-partidos y las estrategias de los partidos políticos, entre otros. A raíz del uso, aplicaciones y replicas metodológicas empleadas por la academia para sus estudios, el MARPOR creó una base de datos complementaria denominada Scope, Range, and Extent of Manifesto Project Data Usage (SRE), siendo esta una base de datos centrada en almacenar las publicaciones que citan el MARPOR, siempre a través del análisis de contenidos. En la actualidad, la SRE almacena 396 publicaciones realizadas entre el año 2000 y el año 2018 en ocho revistas de alto impacto. Es muy representativo que el MARPOR haya aportado a la academia el hecho de validar los planes electorales como fuente de información primaria para el análisis de la ciencia política, dado que este paso puede ser parte del proceso de esa búsqueda de reconocimiento jurídico para dichos documentos. 
Tabla 2

Línea del tiempo de la calidad de la democracia representativa y los planes electorales

Rendimiento de la democracia
El interés por el estudio de la calidad de la de-
mocracia implicó retomar la discusión acerca de
la transición de un régimen político cualquiera a
la democracia. Este debate había generalizado
el concepto de consolidación democrática, y un
grupo de autores (O’Donnell y Schmitter, 1994;
O`Donell, 1996ạ, 1996b, 1997; Günther, R et al.,
1996; Przeworski, 1995, 1998, 1999), basados en
ello, postulan los primeros lineamientos acerca de
la teoría del rendimiento democrático.

En una segunda etapa de construcción del cuerpo teórico para la calidad de la democracia, se identifican diferentes tipos de regímenes, desde el autoritarismo puro a la democracia, y, en medio, regímenes híbridos, entre otros (O’Donnell, 1992; Zakaria, 1998; Mainwaring, Brincks y Pérez-Liñán, 2001; Diamond, 2002; Morlino, 2004, 2005,2007; Mainwaring y Pérez-Liñán, 2008).

De los dos debates anteriores surgen propuestas acerca del concepto y la metodología del cálculo de la calidad de la democracia, y, para ello, arguyen algunos autores, hay que considerar que dicho rendimiento solo puede evaluarse en Estados que tengan las características mínimas del régimen democrático (Lijphart, 1999; Altman y Pérez Liñan, 2002; Dahl, 2002; O’Donnell, 1997; Mazzuca, 2003; Morlino y Diamond, 2005; Vargas -Machuca, 2006; Velasco, 2006; Schmitter, 2005; Morlino 2007).

El debate que está vigente es el concerniente a las dimensiones o variables y métodos que se usan para la medición del rendimiento democrático.

\section{Planes electorales}

Los partidos políticos se estudian desde Hume (1790, traducido por haakonssen, 1993), sin hacer mención a los programas electorales.

Estados Unidos, país pionero en el surgimiento de los partidos políticos, partido demócrata (1828) y partido republicano (1854), referencia plataformas electorales que los diferenciaba en términos de ideas tributarias y antiesclavistas. En Europa se tiene constancia de la existencia de programas electorales desde 1891 (programa del partido social demócrata de Alemania), y en Latino América, por cuenta de México, desde 1906.

El compendio literario acerca de los partidos políticos también dedica un apartado a a la definición y función del plan electoral. En efecto, para Schattschneider (1942) el programa es el resultado del interés de los miembros del partido por sociabilizar su ideología, para Downs (1957) el programa, al igual que los partidos, se presenta con el fin de maximizar votos, y para May (1973) el programa solo refleja los intereses de algunos miembros de los partidos, inclusive afirma que deben existir miembros de esos partidos que no tienen ningún interés en el programa.

A pesar de las referencias anteriores, no es hasta después de la Segunda Guerra Mundial que se inicia la constitución de partidos y sus respectivos programas. Los programas son exigidos, por cuenta de las instituciones electorales, en muchos países para que un partido pueda presentar su candidato.

Finalmente, desde 1979 la academia cuenta con el Manifesto Research on Political Representation (MARPOR), una base de datos que analiza y codifica los manifestos de 1.043 partidos políticos de 60 países, lo cual cubre las elecciones desde 1945. La comunidad científica ha tomado esta base como fuente primaria de información para un sinnúmero de investigaciones que también se pueden consultar en el MARPOR. 
En resumen, el documento plantea que existe una relación vinculante democracia representativa-partidos políticos- programas electorales- calidad de la democracia, y que esa relación permite demostrar la tesis de que los programas electorales influyen en la calidad de la democracia. El argumento que valida esa tesis es que el reconocimiento institucional que las constituciones otorgan a los partidos políticos se traslada implícitamente a los programas electorales, los cuales son contenedores de la información que sustenta la responsabilidad y el compromiso que los representantes gubernamentales asumen cuando ganan una contienda electoral. Además, los planes electorales deben ser vistos como el vínculo que materializa la comunicación entre los partidos y el Estado, relación planteada desde la teoría de la representación democrática, que elige estos estamentos como unidades básicas del régimen democrático.

\section{Conclusiones}

Después de un recorrido teórico amplio, se da por cierta la tesis acerca de la influencia de los programas electorales en el rendimiento de la democracia representativa. No obstante, somos conscientes de que el recorrido necesario para llegar a consolidar esta afirmación no solo es largo sino también controvertido. Como se plantea a lo largo del documento, el primer objetivo se centra en conseguir el reconocimiento jurídico de los programas electorales, hito cuyo éxito quizás depende en gran medida del avance en términos de democratización de todos los actores de la esfera política. Los Estados denominados a través de la democracia representativa como Estados de derecho, los cuales se caracterizan por almacenar en su carta magna las normas y las instituciones a las cuales, entre otros agentes, los representante políticos se deben someter, tienen que hacer explícito el reconocimiento de los programas electorales como documentos legales tanto para validar los compromisos de los representantes políticos como para facilitar la rendición de cuentas de los mismos y de los propios partidos que los sustentan; estos últimos deben asumir con total responsabilidad el contenido presentado en sus programas, debido a que un gran número de votantes se basa en dicho contenido, bien porque lo leen o bien porque se guían por lo que los medios de comunicación trasmiten del mismo, para decidir la orientación de su voto, y a que los costes de elaboración y difusión de esos programas son cubiertos con presupuesto público. Por último, es necesario que los ciudadanos se apropien del contenido de los programas electorales, porque los mismos almacenan las estrategias que los partidos proponen para satisfacer sus demandas y dar cumplimiento a sus expectativas, $y$, por tanto, les han de servir de aval cuando requieran de sus representantes un rendimiento de cuentas adecuado a su oficio político.

Una manera rápida de empezar a trabajar en la legalización de los programas electorales es institucionalizar la evaluación de dichos planes que las entidades electo- 
rales de cada país exigen a cada partido político como oficialización de su inscripción en una contienda electoral. Al respecto, Holanda realiza una evaluación ex-ante y expost a los planes electorales, y aunque el sometimiento a evaluación no es obligatorio, todos los partidos se han vinculado al proceso. Reino Unido, en 2015, como respuesta a la demanda ciudadana de validación de los programas electorales, sometió estos documentos a una evaluación ex-ante, y los partidos debieron responder a los resultados de esa evaluación, defendiendo la validez de sus propuestas. Además de la diferencia notoria de que en Holanda la evaluación es realizada tanto antes como después, también es relevante señalar que, en el primer caso, la evaluación está a cargo de un organismo autónomo, mientras que, en el segundo caso, la evaluación está a cargo del Instituto de Estudios Fiscales. Estos son dos casos que refuerzan la validez de la tesis defendida, dado que demuestran que la legalización del plan electoral depende del grado de democratización de los agentes, y que este documento facilita tanto la accountability como la evaluación de la satisfacción de los electores; en ambos casos, el holandés y el británico, los ciudadanos se interesan por estudiar la viabilidad de los programas presentados por sus candidatos, considerando relevante que en el caso británico, además, fue el mismo pueblo el que solicitó dicha evaluación.

Este documento exige una segunda versión que permita ayudar a consolidar el argumento principal, en la cual, como es lógico, sería interesante valorar la relevancia y la influencia del plan electoral en términos empíricos para la calidad de la democracia. Para ello, se debe pensar en la creación de índices que den cuenta de que los programas realmente son el vínculo entre partidos y electores, y que determinan la decisión de voto. También se pueden crear matrices que den cuenta del cumplimiento de los planes electorales, informando también sobre las diferencias detectadas entre países y territorios diversos. Al respecto, existe un gran compendio de investigadores empíricos que afirman que los programas electorales son documentos válidos para el análisis de la política (Downs,1957; Manin, Przeworski y Stokes, 2001; Körösényi y Sebok, 2013). Otros autores arguyen que las políticas planteadas en los programas electorales son un buen indicador de las políticas que se aplicarán si se ganan las elecciones (Klingemann et al, 1994; McDonald y Budge, 2005). Desde otro punto de vista, algunos investigadores afirman que los partidos políticos de los países europeos cumplen en un 70\% sus promesas electorales (McCluskey, 2008; Rallings, 1987; Thomson, 2001; Bara, 2005; Hofferbert et al., 1994). Todos estos trabajos, a sumar a la solidez aportada por el MARPOR en el mismo sentido, muestran la relevancia del programa electoral, y no única y exclusivamente como herramienta de análisis de los hechos políticos, sino también como documento legal clave en el proceso de evaluación e impulso de la calidad de la democracia, fundamentando y ayudando a consolidar el principal argumento que defiende este artículo. 


\section{Bibliografía}

ALONSO, S., A. VOLKENS, y B. GÓMEZ (2012) Análisis de contenido de textos políticos. Un enfoquecuantitativo, Madrid: Centro de Investigaciones Sociológicas.

ALTMAN, D. y A. PÉREZ-LIÑÁN (2002) "Assessing the Quality of Democracy: Freedom, Competitiveness and Participation in Eighteen Latin American Countries", Democratization, 9 (2), 85-100.

ARES, C., y A. VOLKENS (2017) “¿Por qué y cómo se está extendiendo el Manifesto Project a América Latina?”, Revista Española de Ciencia Política, (43), 115-135.

BARA, J. (2005) “A Question of Trust: Implementing Party Manifestos", Parliamentary Affairs, 58 (3), 585-599.

BEETHAM, D. (1994a) Defining and measuring democracy, Sage, David (ed.).

BOBBIO, N. (1999) "Rappresentanza e interessi”, en M. Bovero (ed.) Teoría Generale della política, Turín: Einaudi, 410-428.

BODINO, Jean (1992) Los seis libros de la república, Selección, traducción y estudio preliminar de Pedro Bravo Gala, Madrid: Tecnos.

COPPEDGE, M. (1990) "Meassuring Poliarchy", Studies in Comparative International Development, 25 (1), 51-72.

COPPEDGE, M. (1994) "Instituciones y gobernabilidad democrática en América Latina”, Síntesis: Revista Documental de Ciencias Sociales Iberoamericanas, (22), 61-88.

COPPEDGE, M. (2002) "Democracy and Dimensions. Comments on Munck and Verkuilen.", Comparative Political Studies, 35 (1), 35-39.

COPPEDGE, M., J.GERRING, D. ALTMAN, M. BERNHARD, S. FISH, A. HICKEN...y H. A. SEMETKO (2011) "Conceptualizing and measuring democracy: A new approach., Perspectives on Politics, 9 (2), 247-267.

DAHL, R. (1971) "La poliarquía", Participación y oposición.

DAHL, R. (2002). La poliarquía: participación y oposición, Madrid: Tecnos.

DIAMOND, L. (2002) “Thinking About Hybrid Regimes.", Journal of Democracy, 13 (2), 21-35.

DIAMOND, L. y L. MORLINO (2005) Assessing the quality of democracy, JHU Press. DIDIER, P. (2000) De la représentation en droit privé, Paris: Librairie Genérale de Droit et de Jurisprudence.

DIGGS, D. J. (1968) "Practical Representation", Nomos, X, 28-37.

DOWNS, A. (1957) An economic Theory of Democracy, Nueva York: Harper \&Row.

DUVERGER, M. (1954) Political Parties: Their Organization and Activity in the Modern State, Londres: Methuen.

ELDERSVELD, S. J. (1964) Political Parties: A Behavioral Analysis, Chicago: Rand McNally. 
EPSTEIN, L. D. (1967) Political Parties in Western Democracies, Nueva York: Praeger. FERRAJOLI, L. (2007) Principia Iuris: Teoria del diritto e della democracia, RomaBari: Laterza\&Figli.

GÜNTHER, R, P. DIAMANDOUROS y H. PUBLE (1996) "Las ilusiones de O’Donnell: una réplica.", La Política: Revista de estudios sobre el estado y la sociedad, (2), 119-128.

HELLER, H. (1998) Teoría del Estado, Traducción de Luis Tobio, edición y prólogo de Gerhart Niemeyer, $2^{a}$ edición en español.

HOBBES, T. (1987) Del ciudadano y Leviatán, $1^{\circ}$ reimpresión, Madrid: Tecnos.

HOFFERBERT, R. I., H. KLINGEMANN, y I. BUDGE (1994) Parties, policies and democracy, Boulder, Colorado: Westview Press.

HAAKONSSEN, K., (1993) The structure of Hume's political theory. The cambridge companion to Hume, vol. 182.

IDEA, Institute for Democracy and Electoral Asistance, Internacional (2002) Handbook on Democracy Assessment, Manual para la evaluación de la democracia, La Haya: Kluwer Law International.

INKELES, A. (1990) "Introduction: On measuring democracy.", Studies in Comparative International Development, 25 (1), 3-6.

JELLINEK, G. (1981) Teoría General del Estado [1900 y 1905], traducción española de la 2. a edición alemana y prólogo de Fernando de los Ríos, Buenos Aires: Editorial Albatros.

KEY, V. y A. HEARD (1949) Southern politics in state and nation, New York: Vintage Books.

KLINGEMANN, H., I. RICHARD y I. BUDGE (1994) Parties, Policies and Democracy, Boulder, CO: Westview.

KÖRÖSÉNYI, A. y M. SEBOK (2013) “The Realistic Version of Positive Mandate Theory. A Political Theory of Pledge-Fulfillment.", MTA TK Politikatudományi Intézet Working Papers in Political Science, 14, 1-21.

LABAND, P., A. R. BEREIJO y J. ZAMIT (1979) Derecho presupuestario, Instituto de Estudios Fiscales.

LAPALOMBARA, J. y M. WEINER (1966) Political Parties and Political Development. (SPD-6), Princeton University Press.

LAPORTA, F. J. (1989) "Sobre la teoría de la democracia y el concepto de representación política: Algunas propuestas para debate", Doxa: Cuadernos de Filosofía del Derecho, (6), 121-141.

LEVINE, D. y J. MOLINA (2007) "La calidad de la democracia en América Latina: una visión comparada.", América Latina Hoy, 45, 17-46. 
LIFANTE, V.I. (2009) “Sobre el concepto de representación”, Doxa: Cuadernos de Filosofía del Derecho, (32), 497-524.

LIJPHART, A. (1999) "Patterns of democracy: Government forms and performance in thirty-six countries.", Yale University Press.

LIPSET, S. M. y S. ROKKAN (ed.) (1967) Party systems and voter alignments: Crossnational perspectives, The Free Press.

LOCKE, J. (2002) Compendio del Ensayo sobre el entendimiento humano, Madrid: Editorial Alba.

MAINWARING, S., D. BRINKS y A. PÉREZ-LIÑÁN (2001) “Classifying political regimes in Latin., Studies in Comparative International Development, 36 (1), 37-65.

MAINWARING, S y A. PÉREZ-LIÑÁN (2008) "Latin American Democratization since 1978., The Third Wave of Democratization in Latin America, New York: Cambridge University Press, 14-59.

MANIN, B., A. PRZEWORSKI, y S. STOKES (2001) Democracy, Accountability, and Representation, Cambridge: Cambridge UniversityPress.

MAQUIAVELO, N. (2010) El príncipe, 204, Ediciones AKAL.

MAY, J. D. (1973) "Opinion structure of political parties: the special law of curvilinear disparity., Political Studies, 21 (2), 135-151.

MAZZUCA, S. (2003) “Calidad democrática: Deber y haber del concepto”, en Osvaldo Iazzeta et al. (Ed.) Democracia, desarrollo humano y ciudadanía. Reflexiones

sobre la calidad de la democracia en América Latina, Rosario-Santa Fe: Homo Sapiens Ediciones-PNUD.

MCCLUSKEY, N. P. (2008) A Policy of Honesty: Election Manifesto Pledge Fulfilment in New Zealand 1972-2005, University of Canterbury.

MCDONALD, M. y I. BUDGE (2005) Elections, Parties, and Democracy: Conferring the Median Mandate, Oxford: Oxford University Press on Demand.

MERRIAM, C. E. (1922) The American Party System, Nueva York: Macmillan.

MICHELS, R. (1962 [1911]) Political Parties: A Sociological Study of the Organizational Tendencies in Modern Democracies, Nueva York: The Free Press.

MONTESQUIEU, B. D. S. (1984) El Espíritu de las Leyes (1748), Edición de Grupo Axel Springer.

MORLINO, L. (2004) “What is a “Good” Democracy., Democratization, 11 (5), 10-32.

MORLINO, L. (2005) Democracias y democratizaciones, México: Centro de Estudios de Política Comparada.

MORLINO, L. (2007) "Explicar la calidad democrática: ¿qué tan relevantes son las tradiciones autoritarias?”, Revista de Ciencia Política (Santiago), 27 (2), 3-22. 
O’DONNELL, G. (1992) “¿Democracia delegativa?”, Cuadernos del CLAEH, 17 (1), 6-20.

O'DONNELL, G. y P. Schmitter (1994) “Transiciones desde un gobierno autoritario.", Volumen 4: Conclusiones tentativas sobre las democracias inciertas. Buenos Aires: Paidós.

O’DONNELL, G. (1996a) “Otra institucionalización. La democratización y sus límites, después de la tercera ola, la política., Revista de Estudios sobre el Estado y la Sociedad, (2).

O’DONNELL, G. (1996b) "Ilusiones y errores conceptuales.", La Política: Revista de estudios sobre el estado y la sociedad, (2), 129-139.

O'DONNELL, G. (1997) Contrapuntos: Ensayos escogidos sobre autoritarismo y democratización, Buenos Aires: Paidós.

O'DONNELL, G., J. VARGAS y O. IAZZETTA (2004) Human Development, Human Rights and Democracy. The Quality of Democracy: Theory and Applications, Notre Dame: University of Notre Dame Press.

NEUMANN, S. (1956) “Toward a Comparative Study of Political Parties", en Neumann (Ed.) Modern Political Parties: Approaches to Comparative Politics, Chicago: The University of Chicago Press.

OSTROGORSKI, M. (1964 [1902]) Democracy and the Organization of Political Parties, Londres: Macmillan.

PITKIN, H. F. (1985) El concepto de representación, Madrid: Centro de Estudios Constitucionales (original inglés de 1967).

PRZEWORSKI, A. (1995) Democracia y mercado, Cambridge: Cambridge University Press.

PRZEWORSKI, A. (1998) Democracia sustentable, Buenos Aires: Paidós.

PRZEWORSKI, A. (1999) “Democracia y representación.", Metapolítica, 3 (10), 227 257.

RALLINGS, C. (1987) "The Influence of Election Programmes: Britain and Canada 1945-1979", en I. Budge, D. Robertson y D. Hearl (Eds.) Ideology, Strategy, and Party Change. Spatial Analysis of Post-War Election Programmes in 19 Democracies, Cambridge: Cambridge University Press.

RANNEY, A. y W. KENDALL (1954) "The American party systems", American Political Science Review, 48 (2), 477-485.

REHFELD, A. (2009) "Representation Rethought: On Trustees, Delegates, and Gyroscopes in the Study of Political Representation and Democracy", American Political Science Review, 103 (2), 214-230.

ROBERTSON, D. (1976) A theory of party competition, Londres: John Wiley and Sons. 
ROUSSEAU, J. J. (1998) Del contrato social: Discurso sobre las ciencias y las artes; Discurso sobre el origen y fundamento de la desigualdad entre los hombres, Madrid: Alianza Editorial.

RUESCHEMEYER, D. (2005) Addressing Inequality, En Diamond, Larry y Leonardo. SARTORI, G. (1976) Parties and Party Systems: A Framework for Analysis, Cambridge: Cambridge University Press.

SARTORI, G. (1999) “Constitución”, Elementos de Teoría Política (1987), Traducción de M. Luz Morán, Madrid: Alianza Editorial.

SCHATTSCHNEIDER, E. (1942) Party government, Transaction Publishers.

SCHMITTER, P. (2005) "Calidad de la democracia: Las virtudes ambiguas de la rendición de cuentas., Metapolítica, (39), 61-73.

SORAUF, F. J. (1964) Political Parties in the American System, Boston: Little, Brown and Company.

THOMSON, R. (2001) "The Programme to Policy Linkage: The Fulfilment of Electoral Pledges on Socio-Economic Policy in the Netherlands, 1986-1998., European Journal of Political Research, 40 (2), 171-197.

VARGAS-MACHUCA, R. (2006) "La calidad de la democracia”, Claves de razón práctica, (165), 34-41.

VELASCO, J. (2006) "Deliberación y calidad de la democracia”, Claves de razón práctica, (167), 36-43.

VOLKENS, A, P. LEHMANN, T. MATTHIEß, N. MERZ, S. REGEL, y B. WEßELS (2018): The Manifesto Data Collection. Manifesto Project (MRG/CMP/MARPOR). Version 2018a. Berlin: Wissenschaftszentrum Berlin fürSozialforschung (WZB). https://doi.org/10.25522/manifesto.mpds.2018a.

VON GERBER, C. F. (1913) "Grundzüge des deutschen Staatsrechts", en B. Tauchnitz (Ed.) Lineamenti di diritto pubblico, 1865, traducción al italiano de P. L. Lucchini, Diritto pubblico, Milán, Giuffrè, 1971, 130-133.

WEBER, M. (1968 [1922]) Economy and Society, Berkeley: The University of California Press.

ZAKARIA, F. (1998) "El surgimiento de la democracia liberal.", BID-Gobierno del Ecuador, Publicación ocasional, Programa de Apoyo al Sistema de Gobernabilidad Democrática. 
